\title{
Accessibility to electronic communication for people with cognitive disabilities: a systematic search and review of empirical evidence
}

\author{
Johan Borg • Ann Lantz · Jan Gulliksen
}

Published online: 19 April 2014

(c) The Author(s) 2014. This article is published with open access at Springerlink.com

\begin{abstract}
The purpose of this study was to identify and synthesize measures for accessibility to electronic communication for people with cognitive disabilities by seeking answers to the following research questions: What measures to make electronic communication accessible to people with cognitive disabilities are evaluated and reported in the scientific literature? What documented effects do these measures have? Empirical studies describing and assessing cognitive accessibility measures were identified by searches of 13 databases. Data were extracted and methodological quality was assessed. Findings were analyzed and recommendations for practice and research were made. Twenty-nine articles with considerable variations in studied accessibility measures, diagnoses, methods, outcome measures, and quality were included. They address the use of Internet, e-mail, telephone, chat, television, multimedia interfaces, texts and pictures, operation of equipment, and entering of information. Although thin, the current evidence base indicates that the accessibility needs, requirements, and preferences of people with cognitive disabilities are diverse. This ought to be reflected in accessibility guidelines and standards. Studies to systematically develop and recommend effective accessibility measures are needed to address current knowledge gaps.
\end{abstract}

Keywords Accessibility - Cognitive disabilities . Communication · ICT · Usability

J. Borg · A. Lantz $(\bowtie) \cdot$ J. Gulliksen

School of Computer Science and Communication, KTH Royal

Institute of Technology, 10044 Stockholm, Sweden

e-mail: alz@csc.kth.se

\section{Introduction}

\subsection{Background}

The importance of accessibility to information and communication in enabling people with disabilities to fully enjoy all human rights and fundamental freedoms is acknowledged by the convention on the rights of persons with disabilities (CRPD). It requires States Parties to take appropriate measures to ensure to people with disabilities access, on an equal basis with others, to information and communications, including related technologies and systems open or provided to the public [1].

People with cognitive disabilities commonly face barriers to electronic communication, such as using the Web and mobile phones [2,3]. Efforts to address these barriers were initiated and solutions proposed. For example, several guidelines and so-called standards were published to guide the development of information and communication technology (ICT)-based products and services to ensure that electronic communication is made accessible to people with cognitive disabilities. A review of 20 guidelines of web accessibility found four design recommendations that at least half of the studied guidelines supported [2]. The remaining 82 design recommendations were each supported by 1-7 guidelines only. The review noted that the guidelines share certain limitations, such as being based on personal opinions of few experts, lacking supporting references, and lacking indications as to whether a particular guideline represents a consensus of researchers or has been derived from a single, non-replicated study. Therefore, it proposes a "move from trial and error to consensus to evidencebased practice" [2, p. 211]. This text intends to contribute to such a shift by identifying scientifically 
evaluated accessibility measures for electronic communication for people with cognitive disabilities.

Accessibility has been defined in numerous ways. Considering its explicit mentioning of cognitive capabilities, the definition used in this work views accessibility as "the extent to which products, systems, services, environments, and facilities are able to be used by a population with the widest range of characteristics and capabilities (e.g., physical, cognitive, financial, social and cultural) to achieve a specified goal in a specified context" [4].

For the purpose of this paper, electronic communication refers to communication by means of ICT-based devices that support communication and has a user interface [5]. Examples of such devices include mobile and smart phones, tablet, laptop and desktop computers, and kiosks. The term "communication" is used for exchange of information between people (e.g., between a journalist and readers) and exchange of information between a user and a system (e.g., between a traveler and a ticketing kiosk). Traditional communication theories build on the model of transferring information between sender and receiver (e.g., [6]), while more recent communication theories view communication as something constructed by two or more people or actors (e.g., [7, 8]).

Cognitive disabilities include cognitive impairments, and difficulties in performing activities and participation due to such impairments. Health conditions and impairments which may result in cognitive disabilities include attention deficit disorder (ADD), attention deficit hyperactivity disorder (ADHD), Alzheimer's disease, aphasia, Asperger's syndrome, autism, dementia, dyslexia, intellectual impairment, mental illness, psychological impairment. People with cognitive disabilities may experience difficulties in electronic communication due to reduced capacity in mental functions, such as orientation, attention, memory, abstraction, organization and planning, experience and management of time, problem solving, language, and calculation $[9,10]$.

The prevalence of cognitive disabilities is uncertain as different health conditions or impairments may be included, and the criteria may vary between countries. In the UK, about $3.7 \%$ of the population reports severe difficulty with day-to-day activities due to their memory, concentration or learning capacities being affected [11]. Similar prevalence figures for severe or complete problems in remembering and concentrating have been reported from Fiji $(3.5 \%)$, India (3.7 \%), Indonesia (2.9\%), Mongolia (4.0\%), and the Philippines (2.4\%) [12]. Regarding specific diagnoses, worldwide prevalence of ADHD is about 5-7 \%, dementia about 5-7 \%, and intellectual disability about $1 \%$ [13-16]. Dyslexia impacts approximately 5-17\% of a population [17]. Not only people with diagnosed dyslexia find reading difficult. About one in five 15-year-olds in the OECD countries do not demonstrate reading skills that will enable them to participate effectively and productively in life [18].

To ensure that this relatively large group of people can participate in the society, the environment, including products and services for electronic communication, needs to be cognitively accessible. Guidelines do exist. However, they appear to lack consistency and their scientific grounding seems uncertain. To support the development of evidence-based standards and guide future work on $\operatorname{cog}$ nitive accessibility to electronic communication, knowledge about scientifically evaluated solutions or measures is a prerequisite. However, a systematic overview of current solutions was not found in the published literature.

\subsection{Aim, objectives, and research questions}

The performed literature review aimed to summarize the current evidence base on measures for cognitive accessibility to electronic communication. Its objective was to identify and synthesize scientifically evaluated and reported measures for accessibility to electronic communication for people with cognitive disabilities. The following research questions were addressed:

- What measures to make electronic communication accessible to people with cognitive disabilities are evaluated and reported in the scientific literature?

- What documented effects do these measures have?

\section{Methods}

A study protocol, a data extraction form and a quality assessment form were developed to ensure a systematic search and review process.

\subsection{Search strategy}

Searches for empirical studies assessing cognitive accessibility measures were performed in 13 web-based databases, see Table 1. Three categories of search termsMedium, Disability and Outcomes-were used in combination, i.e., one term from each of the three categories was required for a hit.

Medium atm, cash machine*, ${ }^{1}$ communication system*, cellphone*, cloud*, computer*, digital*, electronic communication*, electronic device*, ict, information system*, information tech*, information and communication tech*, interface*, internet, ipad*, ipod*, laptop*, mediated com*,

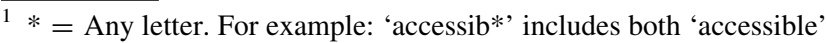
and 'accessibility'.
} 
messaging, mms, mobile phon*, on-line*, pad*, palmtop*, pc, phone*, player*, portable*, reader*, smart card*, smartcard*, smartphone*, sms, social media*, social medium*, surfpad*, tele* (tele communication*, telecommunication*, tele inform*, teleinform*, telephone*, television*), tv, terminal*, text message*, texting, ticket machine*, ticket purchasing point*, vending machine*, video*, web*.

Disability attention deficit, adhd, alzheimer*, aphasia, asperger*, autism, cognitiv* disab*, cognitiv* impair*, communicat* problem*, dementia, development* delay*, difficult* reading, dyslexia, intellectual impair*, intellectual* disab*, language disorder*, language impairment*, learning disab*, learning disorder*, mental* disab*, mental* ill*, mental impair*, mental* retard*, neuropsychia* disab*, neuropsychia* disorder*, neuropsychia* impair*, psych* disab*, psych* impair*, read* difficult*, slow learner*, slow reader*.

Outcome accessib*, comprehen*, effectiv*, effic*, interaction, language*, learnab*, linguistic*, listen*, $\operatorname{read}^{*}$, understand*, usab*, user experience*, usefulness, user friendl*, user satisfaction.

Where possible, searches were limited to abstracts or narrowed using subject specific tools. In Compendex, IEEE Xplore, and Inspec, searches were performed using database-specific terms. In DiVA, searches were performed using two broad search categories, namely, human-computer interaction and interaction technology. ${ }^{2}$

A priori inclusion and exclusion criteria were established. Articles addressing a measure intended to improve access to electronic communication for people with a cognitive disability, reporting primary research and that were peer-reviewed and published 1995 or later were included. Single-case studies, expert opinions, and literature reviews were excluded. The reference lists of selected articles were reviewed for includable studies.

Search terms were identified and agreed by all authors while the searches were carried out by the first author. The total number of hits by database is indicated in Table 1.

The searches were run between February 18 and March 26, 2013 generating a total of 10,206 hits. Applying the inclusion and exclusion criteria, the first review of titles and abstracts resulted in 10,030 hits being excluded. From the remaining 176 hits, 21 duplicates were excluded. The second review of titles and abstracts resulted in the exclusion of 64 articles. Following the review of the full texts of the 91 remaining articles, another 66 articles were excluded, resulting in the selection of 25 articles. An additional 4 articles were included of which one was found in a database and three articles were found in reference lists

\footnotetext{
${ }^{2}$ Contact authors for a complete search history for each database.
}

of selected articles. This resulted in a total of 29 included articles. The search process is summarized in Fig. 1.

\subsection{Quality assessment}

Using an adapted version of a quality assessment tool developed by a health economist [19], the quality of the included articles was assessed in terms of their objective, background, design, methods, data, findings, and discussion, see Appendix. A maximum score of 2 could be awarded to each of 10 items, making the maximum possible total score 20. Quality rating A corresponds to a score of 17-20, B corresponds to a score of 11-16, and C corresponds to a score of 10 or less.

The first author assessed the quality of all included articles and the second author assessed five articles. Any

Table 1 Number of hits by database

\begin{tabular}{lr}
\hline Database & \multicolumn{1}{c}{ Hits } \\
\hline ACM digital library & 0 \\
AMED, CINAHL, and & 1,367 \\
ERIC & \\
BioMed central & 219 \\
Compendex & 1,331 \\
DiVA & 438 \\
IEEE Xplore & 967 \\
Inspec & 873 \\
PubMed & 3,618 \\
ScienceDirect & 96 \\
Scopus & 341 \\
Web of science & 956 \\
Total (13 databases) & 10,206 \\
\hline
\end{tabular}

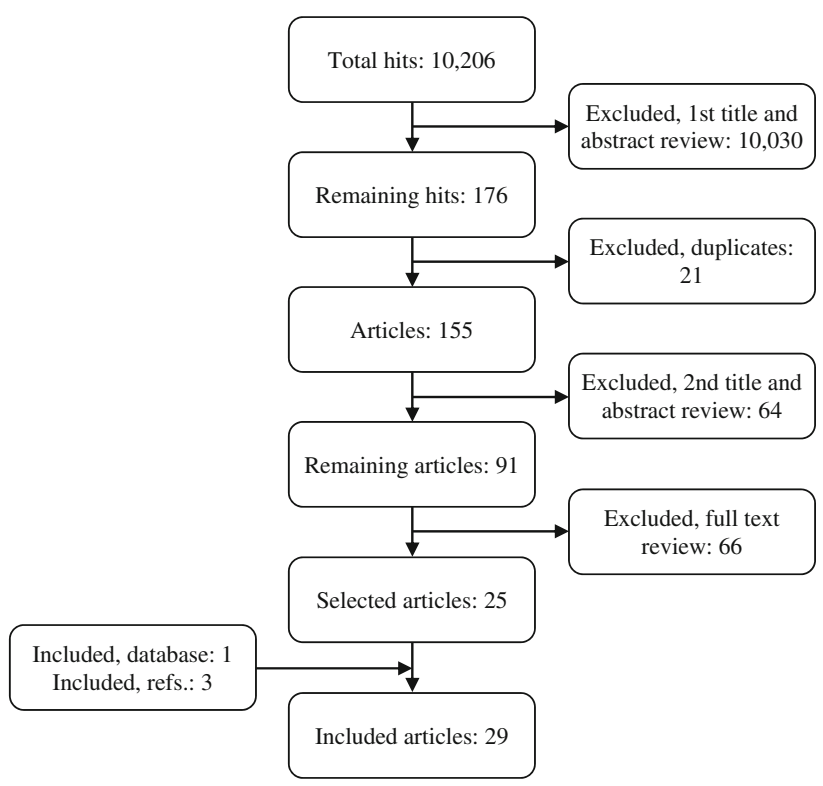

Fig. 1 Flow diagram of study selection 
differences in scoring were discussed by the two authors until consensus was reached. Where necessary, the quality assessments of the other articles were adjusted to reflect the agreed view.

\subsection{Data extraction}

To extract data from included articles, a form comprising the following data extraction categories was developed: Reference, Objective, Design, Method, Number of participants, Disability, Age and gender, Country, Environment, Medium or equipment, Type of communication, Outcome measures, and Results. Data were extracted by the first author. To further summarize the data, the categories were collapsed into broader areas.

Disability terminology evolves, which has resulted in certain terms used in the included articles being obsolete. In addition, preferred terms may vary between countries and disciplines. It is beyond the scope of this review to harmonize the diagnoses. However, the terms "mental retardation" and "developmental cognitive disability" have been replaced by "intellectual disability" except among the search terms and in Table 4, where the original terms remain in brackets.

\subsection{Analysis and discussion}

Extracted data were analyzed thematically according to type of electronic communication with a narrative summary of each included article. The findings are discussed and interpreted in Sect. 4, while implications for practice and research are discussed in Sect. 5.

\section{Results}

\subsection{Description of included articles}

Summaries of countries, reported diagnoses, and types of communication or interaction covered by the 29 included articles are given in Tables 2 and 3. It may be noted that 15 of the articles are based on studies in two countries (USA

Table 2 Countries represented in the articles

\begin{tabular}{ll}
\hline Countries & $\begin{array}{l}\text { No. of } \\
\text { articles }\end{array}$ \\
\hline USA & 9 \\
UK & 6 \\
Spain & 3 \\
France & 2 \\
Belgium, Finland, Germany, Israel, Italy, Japan, & 1 \\
$\quad$ Netherlands, Norway, Taiwan & \\
\hline
\end{tabular}

and UK) while the remaining 14 articles present studies from 11 different countries. The most common diagnoses in the articles were intellectual disability (eight articles) and dyslexia (six articles). The most common type of communication or interaction was Internet and reading texts on screen (five articles each).

A brief summary of each article is given in Table 4 . Fourteen articles got quality rating A, 12 articles got B, and 3 articles got $\mathrm{C}$.

\subsection{Narrative synthesis}

In this sub-section, a narrative synthesis of the reviewed articles is provided based on their type of communication or interaction. Accessibility measures and their outcomes among studied disability groups are described.

\subsubsection{Internet}

The use of web browsers and sites were studied in five articles. Compared with using Internet Explorer, users with intellectual disability performed statistically significantly better when they used a web browser that included audio prompting, reduced screen clutter, personalization and customization, graphics, consistent placement of buttons, and automating steps. Two types of audio prompting were used: (1) a message describing the use of a button was played when the cursor arrow was placed over it; (2) a message guiding the user to the next-most-likely step was played after a user-initiated event. A minimum of buttons and on screen features were displayed to minimize screen clutter [20]. In another comparison involving Internet Explorer, an adapted web browser increased reading comprehension among children with intellectual disability to a statistically significant degree. The adaptations included modification of the toolbar with functions used most frequently in Internet Explorer, voice description for toolbar functions, reading out of highlighted words or sentences by synthetic speech, and automatic pop-up of pictures corresponding to words or phrases when the user moves the mouse over them [21].

A comparison of a conventional website and an adapted version of it reported significant improvements in terms of usability and satisfaction among users with intellectual disability. The adapted website featured web pages that could be visualized in a full-screen format, elimination of the browser menu and controls, elimination of scrolling, back and home buttons inside the web pages, descriptive texts at the top of the pages, audio instructions, options represented by pagecentered images distributed around a selection pictogram, and structured step-wise navigation [22]. In another comparison, this time between a standard website and a website developed for people with early-stage dementia, users with 


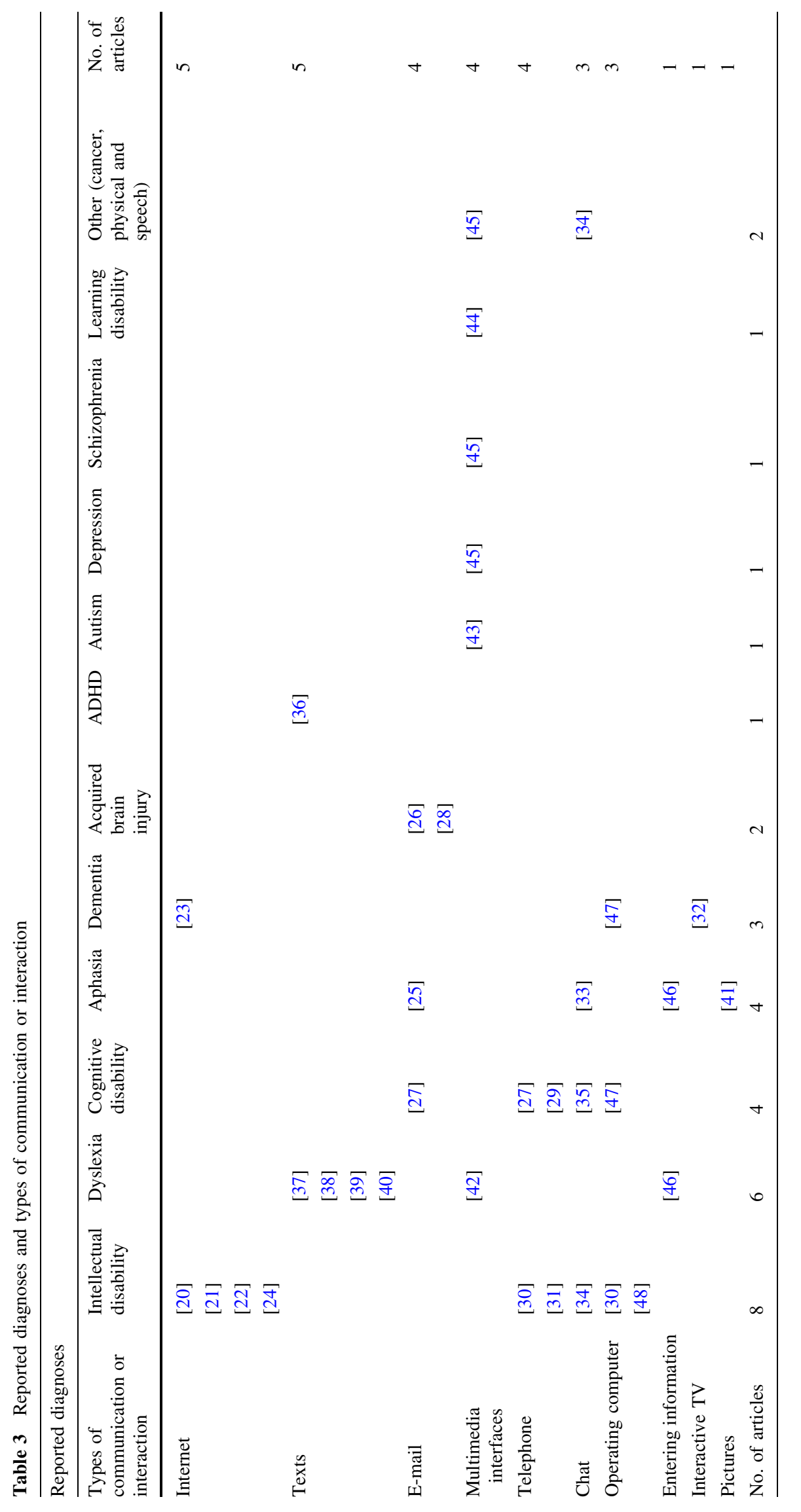




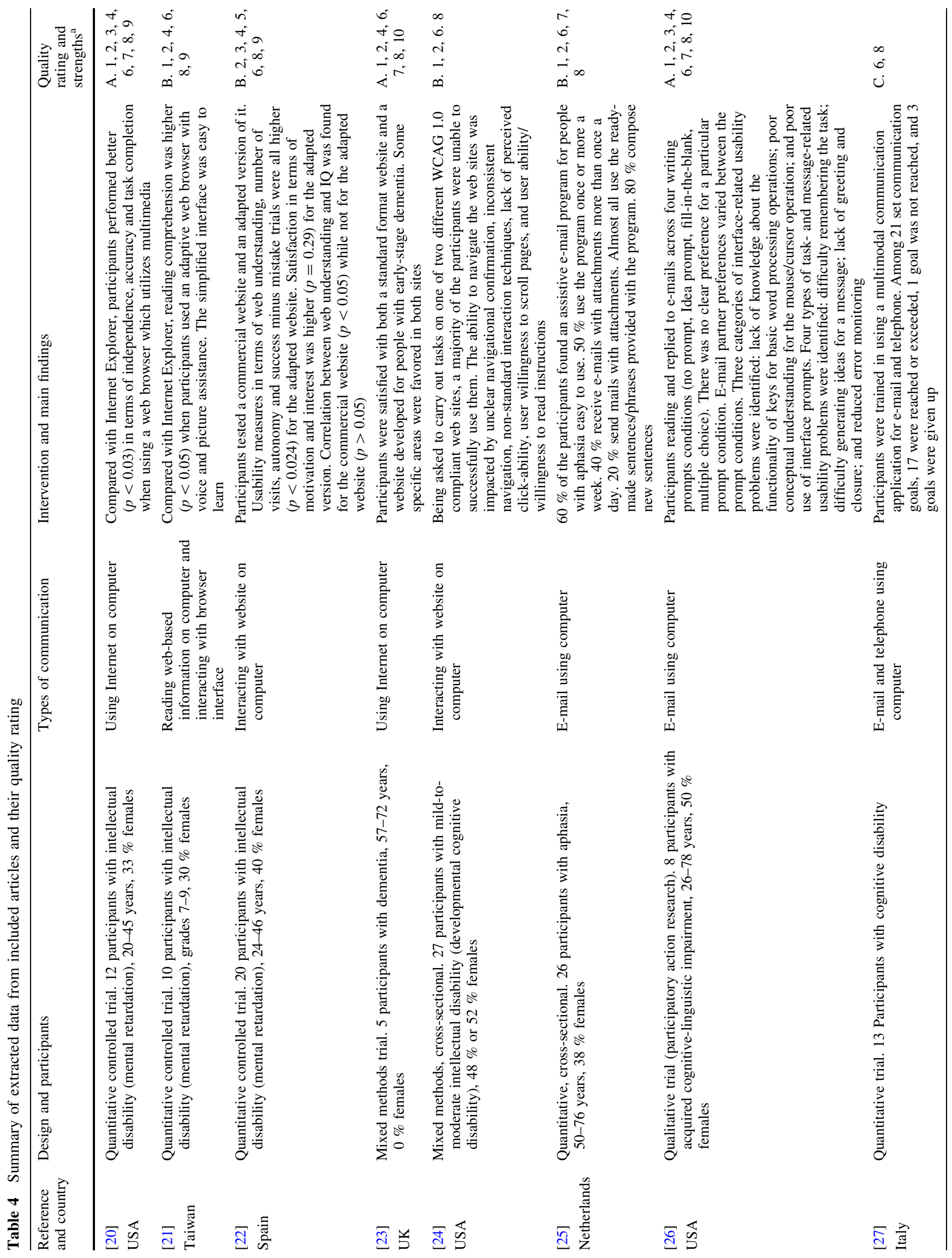




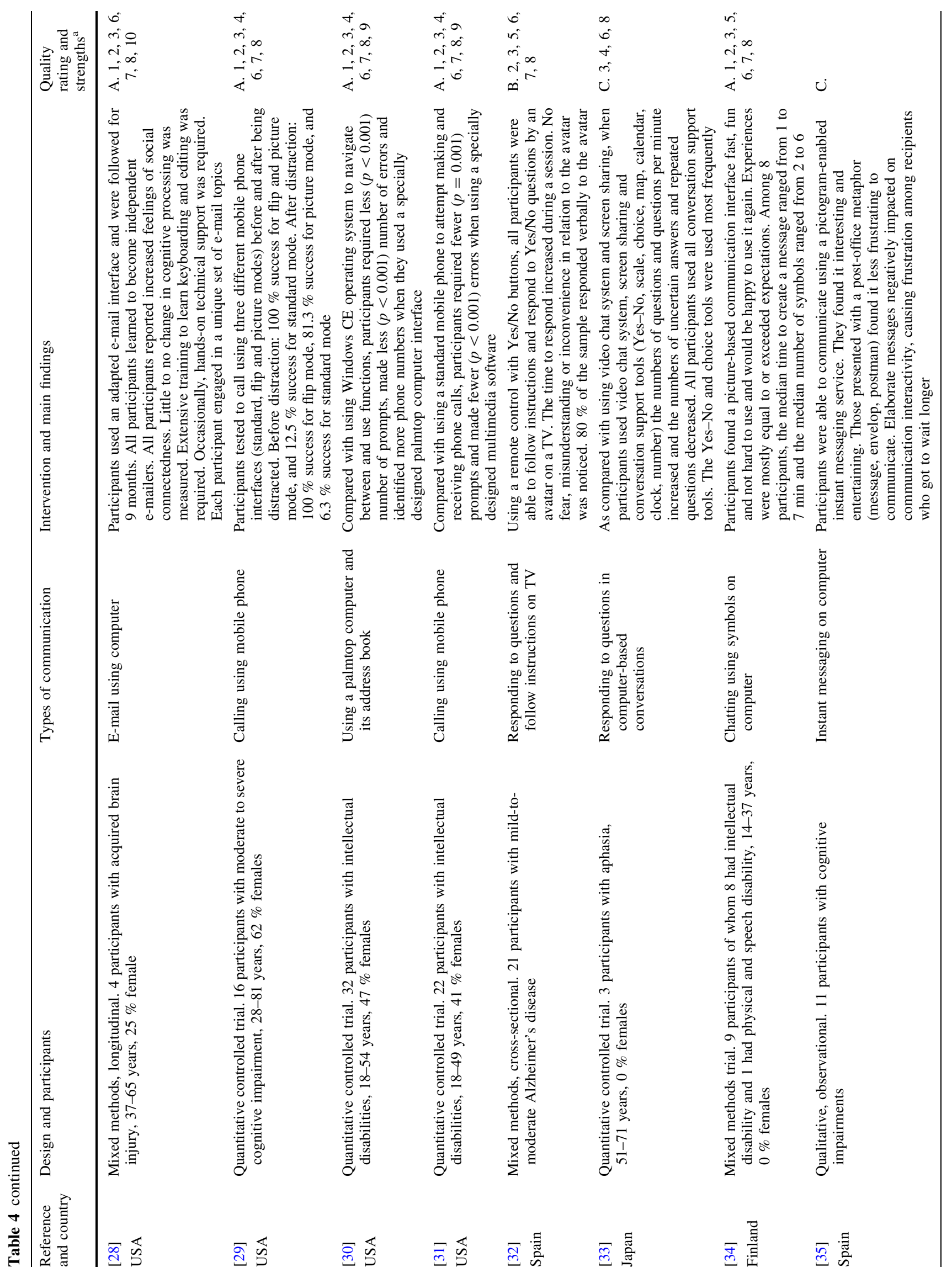




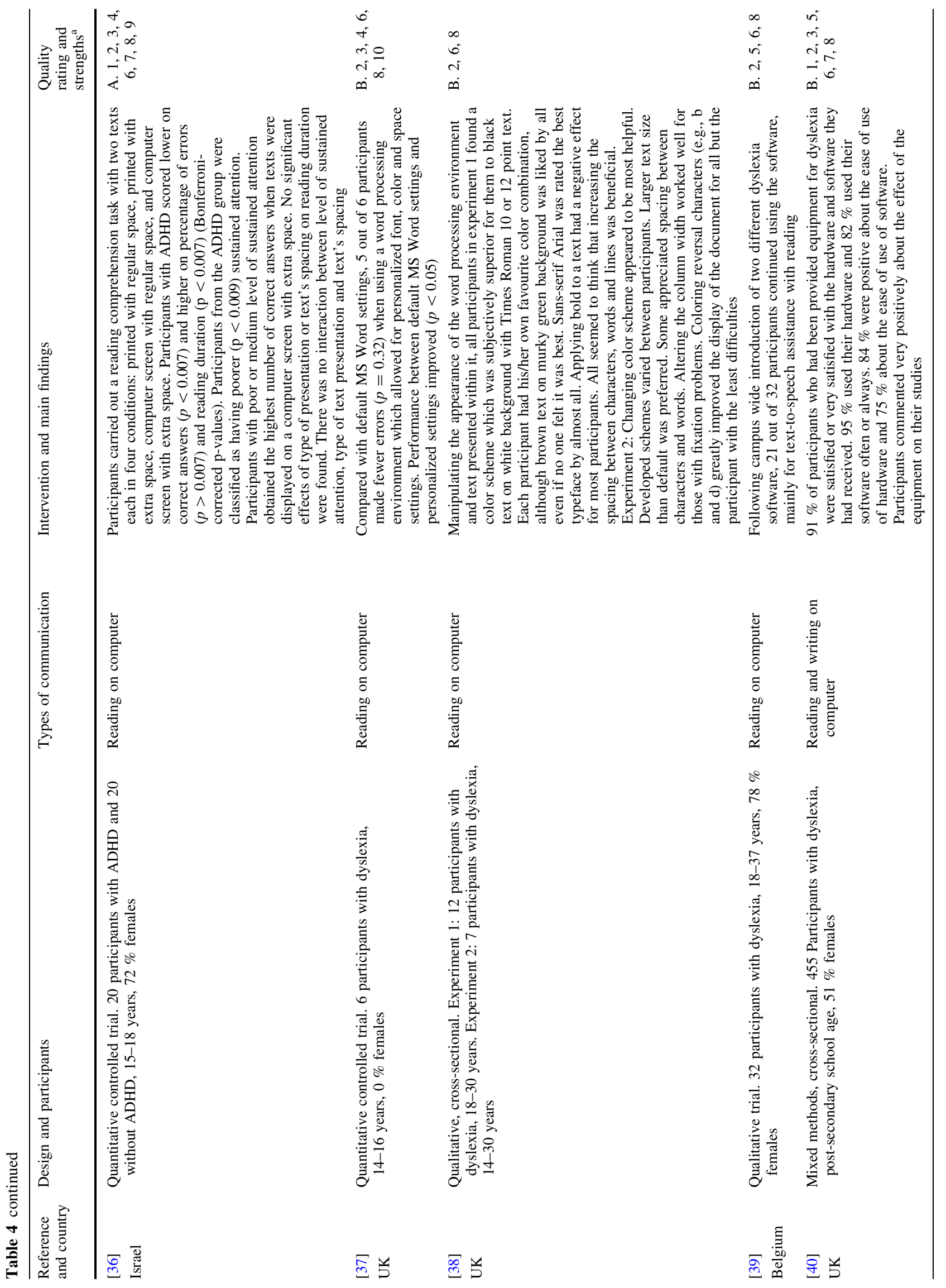




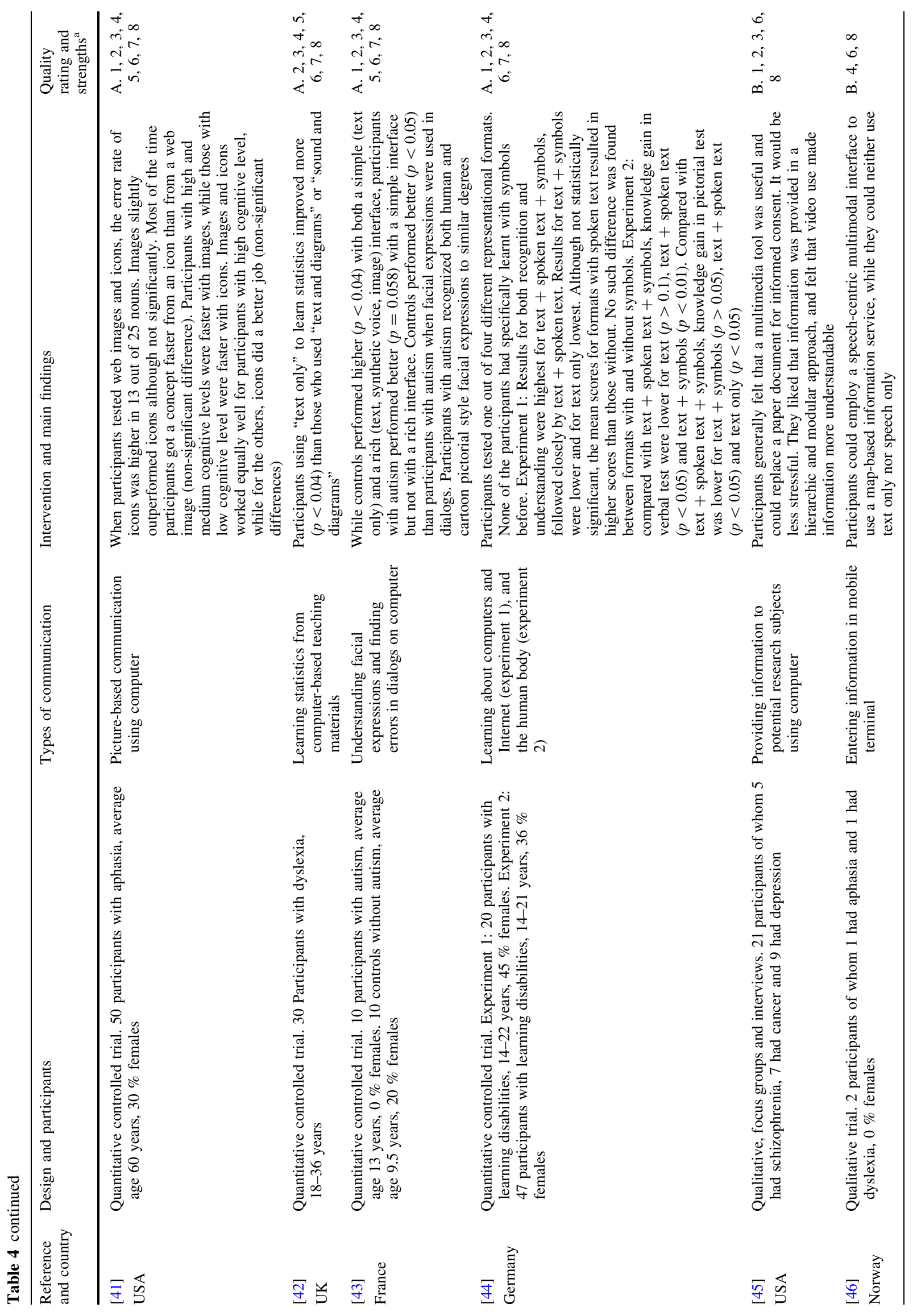




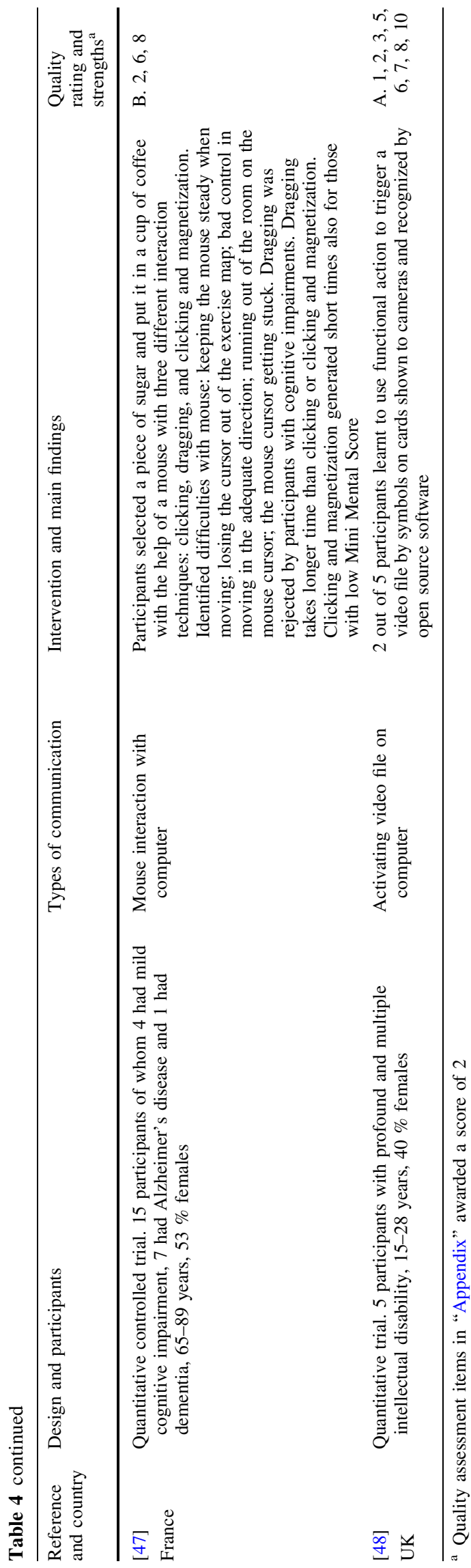

dementia were satisfied with both sites but preferred different features of them. Although the length of the pages was not shorter on the new website, users experienced fewer scrolling problems on it, which is suggested to be explained by "cleaner" look of the pages and fewer choices. With changes to the contents menu, particularly with the addition of icons, there was a greater confusion between menu and text on the new site. There were more instances of clicking on nonlinked items in the new site, mainly on explanatory bullet points [23].

In a study of two WCAG 1.0 compliant web sites, users with intellectual disability were unable to use both of them [24].

\subsubsection{E-mail}

Four articles address electronic communication via e-mail. An e-mail program with categorized ready-made sentences or phrases and images that can be included in the mail was found to be easy to use by $60 \%$ of study participants with aphasia while the remaining participants found the program reasonably simple or complex to use [25]. In a comparison of four prompt conditions to write e-mails, the participants with cognitive impairments did not express any clear preference for writing on a blank e-mail screen, writing on a blank e-mail screen below a list of e-mail composition ideas, filling in the blanks in an e-mail template by writing, or filling in the blanks in an e-mail template by choosing words from pull-down menus with five set options [26]. In addition to composing mails using texts and icons, one e-mail program allowed recorded speech and the use of customizable keyboards. Although most of the 21 users with cognitive disabilities appeared to have achieved or exceeded their goals in using this program, explicit outcomes of it were not reported [27].

In a longitudinal study of a specialized e-mail program, all four users with acquired brain injury endorsed the social benefits of e-mail and achieved successful outcomes for several of their individual goals, including learning a new skill, and feeling connected with friends and family. Features of the e-mail program include that the user cannot exit it, the e-mail partners are fixed and can only be changed by care providers, and the most recently received message from a partner is shown in the top window while the bottom window is used to compose a reply. To overcome problems related to e-mail addresses, a visual address book was implemented [28]. A visual address book was also tested in another e-mail program [27].

\subsubsection{Telephone}

Four articles report on studies of telephone functions. Three of them compare specially designed interfaces with 
standard interfaces, concluding that the former are more effective than the latter. First, three modes of mobile phone operation were tested by participants with moderate to severe cognitive impairment before and after being distracted. Participants were completely successful when they simply had to open the mobile phone (flip mode). When they had to open the mobile phone and touch the correct picture (picture mode), the participants were completely successful before distraction and slightly less successful after distraction. Finally, when they had to dial a 10-digit number and press the send button (standard mode), the success rate was low [29]. Second, in a comparison of Windows CE and a specially designed palmtop computer interface (see description in Sect. 3.2.8), participants with intellectual disabilities were able to correctly identify more phone numbers when they used an interface with pictures [30]. Third, in a comparison of a standard mobile phone and a specially designed interface, participants with intellectual disabilities were more successful in making and receiving phone calls when they used the special interface with, e.g., pictures, audio prompts, and fewer buttons [31].

In a non-controlled trial of a multimodal communication application, participants with cognitive disability also used a visual phonebook. A click on the photo of a person allowed the participants to choose between phone call and e-mail/SMS composition. Phone book scrolling could be manual or automatic. Although no explicit outcomes of using the telephone function are reported, most participants seem to have reached or exceeded their goals [27].

\subsubsection{Interactive $T V$}

Addressing interactive TV, one article reports that users with mild-to-moderate Alzheimer's disease were able to follow instructions and respond to Yes/No questions by an avatar on a TV. Responses were made with a remote control. The avatar had a realistic voice and its lip movements were synchronized with its speech [32].

\subsubsection{Chat}

Three articles on chatting were identified. One controlled trial presents a video chat system with screen sharing complemented with conversation support tools [33]. The conversation support tools increased chat performance of users with aphasia and included:

- Yes-No tool: Window containing "Yes", "No" and "Not understood" buttons.

- Scale tool: A scale bar is shown.

- Choice tool: Text areas for a conversation partner to type in.

- Map tool: Web-based map system.
- Calendar tool: A blank calendar.

- Clock tool: Clock without hands.

- Number tool: A group of numbers.

Two studies explored picture-based communication interfaces, which were found to be fast, fun, not hard to use, interesting, and entertaining. In the first study, participants-mainly with intellectual disabilities - tested a user interface which was organized into three main sections: (1) message history and chat partners, (2) symbol input view, and (3) symbol category view. The application supported various input and output modalities. It was designed for graphical symbols, speech output, and touch-screen input. Text output, mouse interaction, and keyboard input could also be used [34]. In the second study, participants with cognitive impairments were able to communicate using an interface that was divided into a login window, a contacts window, and a dialog window. Written language was replaced by pictograms, including passwords. The dialog window was made up of five sections: (1) pictogram categories, (2) most frequent pictograms in a conversation, (3) pictograms of selected category, (4) actual conversation, and (5) pictogram input space [35].

\subsubsection{Texts}

Five articles reported studies of reading on screens. Noting that participants with ADHD had poorer sustained attention, a controlled trial found that those with poor or medium level of sustained attention obtained the highest number of correct answers in a reading comprehension task when texts were displayed with extra space on a computer screen (see Table 4 for details) [36]. Another controlled trial found that participants with dyslexia made fewer reading errors when they could use personally preferred settings in terms of font, color, and space as compared with MS Word standard settings [37]. This confirms previously published findings of another word processing environment experiment. By altering font, color, and space settings, participants with dyslexia were able to find preferred nonstandard settings (see Table 4 for details). Brown text on murky green background was liked by all even if no one felt it was best. Almost all rated sans-serif Arial as the best typeface [38].

Two studies indicated that software and hardware for people with dyslexia were continuously used by a majority of those who had received them and that they were largely satisfied with equipment $[39,40]$.

\subsubsection{Pictures}

Pictures were studied in one article. In a sample of people with aphasia, it was identified that images and icons 
worked equally well in terms of accuracy for participants with high cognitive level, while for participants with medium or low cognitive level, icons worked better. It was also found that participants with high or medium cognitive level were faster with images, while participants with low cognitive level were faster with icons [41].

\subsubsection{Multimedia interfaces}

Possible benefits of multimedia interfaces have been reported in four articles. In two controlled trials, participants with dyslexia and autism performed better when they used text only interfaces. Students with dyslexia using "text only" to learn statistics improved more than those that used "text and diagrams" or "sound and diagrams" [42], and children with autism performed poorer when they used richer multimedia interfaces (text, speech, and images) while their performance improved when they used a simple interface (text only) [43]. Contrary findings have been reported among participants with learning disabilities. Also in a controlled trial, interfaces with text, spoken text, and symbols resulted in better recognition, understanding and knowledge gain than interfaces with text and spoken text, text and symbols, or text only [44].

In a study in which about two-third of the participants had depression or schizophrenia, a multimedia presentation of information with video was found to improve understandability of informed consent content. Using the system would be less stressful as it gave the participants a greater sense of control [45].

\subsubsection{Entering information}

One article explores a multimodal interface for small mobile terminals, which converts a web service to a mapbased service supporting speech, graphic/text, and pointing modalities as inputs. Two participants with dyslexia and aphasia, respectively, could use the service by pointing at a map while uttering simple commands. They could use it neither by speaking and taking notes in the telephone-based service, nor by writing names in the text-based web service [46].

\subsubsection{Operating equipment}

Operation of equipment was studied in three articles. In comparison with Windows CE operating system for palmtop computers, participants with intellectual disabilities required statistically significantly fewer numbers of prompts and made statistically significantly less number of errors when they used a specially designed palmtop computer interface. The physical buttons on the front of the unit redirected to the new system when pressed, and access to the controls on the Windows Start bar and at the bottom of the display were removed. The new system provided a capability to create customized, oversized multimedia buttons to launch applications and features. Clicking once on a button on the main display generated an audible message identifying the purpose of the button and cuing the user as how to proceed. Tapping a button twice would start an application [30].

Three ways of interacting with a mouse were tested by people with various cognitive disabilities. The "dragging" technique was rejected by the study participants and required more time than "clicking" and "clicking and magnetization" (see Table 4 for further details). Dragging corresponds to the usual drag and drop by maintaining pressure while moving an item on the screen. Clicking corresponds to clicking both at selecting and deselecting an item on the screen item. Finally, clicking and magnetization corresponds to selecting an item by clicking it. It will then follow the cursor until it is moved to a place on the screen where it is deselected automatically [47].

In a study of activating video files by symbols on cards shown to cameras and recognized by open-source software, two of five participants with profound and multiple intellectual disabilities learnt to activate the video file [48].

\section{Discussion}

This section discusses characteristics of the included articles, their findings, and methodological aspects of this review. Implications for practice and future research are also considered.

\subsection{Studies}

Slightly more than half of the articles (15 out of 29) reported studies that were carried out in two countries only. According to cognitive theories, social factors may be of relevance to the design of accessible interfaces for electronic communication for people with cognitive disabilities [49]. Therefore, studies from a wider range of countries or cultures would be required before global accessibility recommendations are made.

Nearly half of the articles (14 out of 29) were limited to two different diagnoses. Each combination of reported diagnoses and types of communication or interaction in Table 3 was covered by very few studies, if any. Considering the role electronic communication plays in contemporary society, this raises a general concern about the attention the scientific community has paid to accessibility to electronic communication for people with cognitive disabilities. This situation calls for initiatives that explore accessibility measures for this group in various settings, as 
appropriate means, modes and formats of communication may vary across diagnoses and social contexts.

Almost half of the reported studies (14 out of 29) had a controlled design, either self-controlled or with a control group. None of the studies reported a power calculation, making it difficult to determine whether the sample sizes were appropriate for the stated purposes. In fact, several studies used small samples. This was reflected in the quality ratings, in which nearly half of the articles (14 out of 29) got the highest rating while 4 articles got the lowest rating. Some articles tended to describe the technical solutions well while other articles described the participants well. A sound discussion of the methodological limitations and their implications were missing in several articles.

The variation in quality rating is partly explained due to differences in type of publication and related limitations in space and differences in type of studies, e.g., pilot studies may be less detailed and not allow for statistical inference.

\subsection{Study findings}

Three controlled studies explored accessibility to Internet browsers and web sites. They present a range of features that reportedly improve the accessibility for users with intellectual disability. Two studies addressed accessibility to web sites. One of them noted that conformance with the accessibility standard web content accessibility guidelines (WCAG) 1.0 did not result in users being able to use such web sites. This may not be unexpected as elements relating to cognitive disabilities have been assigned lower priorities in the WCAG 1.0 [2]. There are concerns that this priority setting largely remains in the updated WCAG 2.0 [50]. In fact, the WCAG 2.0 acknowledges that "content that conforms at the highest level... will not be accessible to individuals with all types, degrees, or combinations of disability, particularly in the cognitive language and learning areas" [51].

Little evidence is available on what measures make e-mail accessible. None of the included articles used a controlled design. However, there are indications that ready-made sentences or phrases facilitate communication of people with aphasia. Moreover, it appears that users with acquired brain injury benefit socially from using a simplified e-mail program.

The use of pictures for making and receiving phone calls is supported by three controlled and one non-controlled trials. Although contemporary mobile phones and smartphones may not have incorporated all studied accessibility features, such as audio prompting and reduced numbers of buttons, most of them allow for pictures of contacts.

Chatting is a form of electronic communication that may or may not allow users to see each other during a conversation. In a controlled trial, ready-made tools for responding were found to improve video chatting performance by people with aphasia. Among other user groups, two studies support the use of symbols and pictograms for chatting.

Reading texts on screens is a common feature of electronic communication. One qualitative and two controlled trials found that performance of users with ADHD and dyslexia improved when texts were displayed with extra space. In addition, users with dyslexia also benefitted from personally preferred fonts and colors. For example, all participants in one study preferred brown text on murky green background over black text on white background. This contradicts sweeping guidelines, such as: "Contrast ratio should be maximized when selecting colors for background and foreground elements" [5]. Rather, evidence indicates that measures to allow for individually selected background and foreground colors should be recommended in order to accommodate the needs of those who require high contrast, e.g., people with visual impairments, as well as the needs of those who benefit from color combinations with lower contrasts.

The importance of allowing for individually preferred settings is underscored by findings from three controlled trials related to multimedia interfaces. Users with dyslexia or autism performed better when they used simpler interfaces while users with learning disabilities performed better with richer multimedia interfaces. As cited by existing web design guidelines for users with cognitive disabilities, the top design recommendation was to use pictures, icons, and symbols along with text [2]. Considering the evidence, it may be better to recommend that the user should be able to set his or her preferred combination of such features.

Although supported by only one controlled trial, the performance of users of varying cognitive levels in using icons and images stresses the importance of allowing for individual preferences.

The identified articles provide little guidance on how to enter information into a system, although a limited test of an innovative multimodal system was reported. Another innovative interface used an avatar on a TV set in combination with a simple remote control, which reportedly worked well for the study participants.

Operating hardware constitutes an important part of electronic communication. One controlled trial indicated that palmtop computer interfaces may be designed in ways that improve performance. Another controlled trial found that drag and drop as a way to interact with a mouse was inefficient for users with cognitive disabilities.

\subsection{Methodological aspects}

Potential limitations of a systematic search and review include possible gaps in the searching procedure. To 
minimize this, a relatively wide range of both specific and general search terms were combined and used in relevant engineering, education, and healthcare databases available to the authors through the library services of two universities.

Not all articles reported diagnoses in a consistent manner, which made it impossible to categorize them properly. It is therefore likely that the diagnoses in Table 3 overlap each other. It would be beneficial if authors use established terminology for health conditions and impairments, e.g., diagnoses or functioning as found in ICF, ICD-10, or DSM-IV [9, 52, 53].

Similarly, the types of communication and interfaces used in Table 3 are based on categories emerging while analyzing the articles. In future work, developing a complete set of categories in advance will facilitate the identification of additional gaps.

For many years, the theoretical approach to communication has been the sender-receiver model. Lately, there has been a shift toward a more constructive approach. The latter leads to an increased demand on the user interface and the importance of its usability and use worthiness, which is of great importance when it comes to support civil rights, i.e., in the context of this article, enabling communication for people with cognitive disabilities.

The fact that about half of the included articles were based in two countries does not necessarily imply that more research in this area is being done there compared with other countries, Therefore, considering the limitation of restricting the review to peer-reviewed and scientifically published articles, our research group undertakes a similar review of gray literature.

Accessibility features of recent technologies and applications, such as tablet computers, smart phones, social networks, and alternative messaging platforms, were not well covered by the included articles. Similarly, possible advancements in terms of accessibility to electronic communication by international project, e.g., AEGIS and Cloud4All, were not considered [54, 55].

\subsection{Implications for practice}

The identified evidence base is rather thin and provides limited guidance for practice. However, it does indicate specific measures that may contribute to making Internet browsers, web sites, texts on screens, calling and certain hardware more accessible to certain groups of people with cognitive disabilities. The most important implication for practice, though, may be that the findings suggest that accessibility measures need to be adaptable at both group and individual levels.

Contrary to the intentions of existing accessibility guidelines, the findings indicate that guidelines conformance may sometimes sustain barriers and thus prevent certain groups from communicating electronically. It is therefore important that available evidence is considered when developing or revising guidelines and standards, such as the WCAG and its accommodation of the accessibility needs of people with intellectual disabilities.

\subsection{Implications for future research}

The findings warrant further research that contributes to creating a sound scientific basis for developing and implementing appropriate accessibility measures. This may include welldesigned replications of some of the presented studies in order to verify or reject their findings. As resources are limited, coordinated efforts to identify, prioritise, and address knowledge gaps in terms of combinations of diagnosis groups and types of communication and interaction may be a cost-effective way forward. Compared with Table 3, the diagnosis as well as means, modes, and formats of communication and interfaces need to be expanded to ensure that all possible aspects are covered. Research priorities should be set in consultation with concerned user groups.

\section{Conclusion}

The findings of this review lead to the following conclusions:

1. The current evidence base on measures for cognitive accessibility to electronic communication is rather thin. Few studies, often with few participants, have researched few types of communication and interaction for a limited number of cognitive diagnoses, making it difficult to generalize most of the reported findings to larger populations.

2. The accessibility needs, requirements, and preferences of people with cognitive disabilities are diverse. Therefore, measures to ensure accessibility to electronic communication need to be individually adaptable. Guidelines and standards ought to reflect this in their recommendations.

3. There is a need for further research in this field, particularly as accessibility to information and communication is a key to people with cognitive disabilities being able to enjoy their human rights and fundamental freedoms.

Acknowledgments This work was funded by the Swedish Post and Telecom Authority (PTS). The need for the study was identified by Torbjörn Lundgren of the Dyslexia Federation FMLS, Sweden, and Hans Hammarlund of FUB, the Swedish National Association for Persons with Intellectual Disability. We are grateful for valuable comments provided by the reviewers. 
Open Access This article is distributed under the terms of the Creative Commons Attribution License which permits any use, distribution, and reproduction in any medium, provided the original author(s) and the source are credited.

\section{Appendix: Quality assessment tool}

To each of the following 10 items, a maximum score of 2 points was awarded. $2=$ Article complies completely. $1=$ Article complies partly. $0=$ Article does not comply at all.

1. Does the study have a clear and well-defined research problem?

2. Does the study justify the research problem?

3. Does the study clearly describe the methods used to address the research problem?

4. Does the study use an experimental design, including controls, to explore causality (maximum score 2)? Does the study use a correlation design to predict outcomes (maximum score 1)? Does the study use a descriptive design to describe and observe relations (maximum score 1)?

5. Are data clearly described with regards to source, collection method, sampling, sample size, time period and level?

6. Are primary data used in the main analyses?

7. Does the study answer all research questions?

8. Are all reported findings and results outcomes of the applied methods?

9. Does the study establish convincing causality between studied causes and effects?

10. Does the study critically discuss possible bias, robustness of the findings and limitations of the method?

\section{References}

1. UN: Convention on the Rights of Persons with Disabilities. Resolution 61/106. United Nations, New York (2007)

2. Friedman, M.G., Bryen, D.N.: Web accessibility design recommendations for people with cognitive disabilities. Technol. Disabil. 19, 205-212 (2007)

3. Bryen, D.N., Carey, A., Friedman, M.: Cell phone use by adults with intellectual disabilities. Intellect. Dev. Disabil. 45, 1-2 (2007)

4. Persson, H., Åhman, H., Arvei Yngling, A., Gulliksen, J.: Universal, inclusive, accessible, design for all; Different conceptsone goal? On the concept of accessibility-historical, methodological and philosophical aspects. Univ. Access. Inf. Soc. (2014). doi:10.1007/s10209-014-0358-z

5. SI, E.T.: Human Factors (HF); Guidelines for ICT Products and Services; "Design for All". European Telecommunications Standards Institute, Sophia Antipolis Cedex (2009)
6. Dimbleby, R., Burton, G.: More Than Words: An Introduction to Communication. Taylor and Francis, Oxon (1998)

7. Wertsch, J.V.: Voices of the Mind: A Sociocultural Approach to Mediated Action. Harvester Wheatsheaf, London (1991)

8. Clark, H.H.: Using Language. Cambridge University Press, Cambridge (1996)

9. WHO: International Classification of Functioning, Disability and Health (ICF). World Health Organization, Geneva (2002)

10. Scherer, M.J., Federici, S., Tiberio, L., Pigliautile, M., Corradi, F., Meloni, F.: ICF core set for matching older adults with dementia and technology. Ageing Int. 37, 414-440 (2012)

11. ODI: Disability prevalence estimates 2010/11. Office for Disability Issues. http://odi.dwp.gov.uk/docs/res/factsheets/dis ability-prevalence.pdf (2012)

12. Mont, D.: Measuring Disability Prevalence. SP Discussion Paper No. 0706. World Bank, Washington (2007)

13. Willcutt, E.G.: The prevalence of DSM-IV attention-deficit/ hyperactivity disorder: a meta analytic review. Neurotherapeutics 9, 490-499 (2012)

14. Polanczyk, G., Silva de Lima, M., Horta, B.L., Biederman, J., Rohde, L.A.: The worldwide prevalence of ADHD: a systematic review and metaregression analysis. Am. J. Psychiatry 164, 942-948 (2007)

15. Prince, M., Bryce, R., Albanese, E., Wimo, A., Ribeiro, W., Ferri, C.P.: The global prevalence of dementia: a systematic review and metaanalysis. Alzheimers Dement 9(1), 63-75.e62 (2013)

16. Maulik, P.K., Mascarenhas, M.N., Mathers, C.D., Dua, T., Saxena, S.: Prevalence of intellectual disability: a meta-analysis of population based studies. Res. Dev. Disabil. 32(2), 419-436 (2011)

17. McCandliss, B.D., Noble, K.G.: The development of reading impairment: a cognitive neuroscience model. Ment. Retard. Dev. Disabil. Res. Rev. 9, 196-204 (2003)

18. CD, O.E.: PISA 2009 Results: Executive Summary. The Organisation for Economic Co-operation and Development, Paris (2010)

19. Ekman, B.: Community-based health insurance in low-income countries: a systematic review of the evidence. Health Policy Plan 19, 249-270 (2004)

20. Davies, D.K., Stock, S.E., Wehmeyer, M.L.: Enhancing independent internet access for individuals with mental retardation through use of a specialized web browser: a pilot study. Educ. Train Ment. Retard. Dev. Disabil. 36, 107-113 (2001)

21. Chu, C.N., Chen, M.C., Li, T.Y.: A study on the design and evaluation of an adaptive web browser for students with reading difficulties. In: ICCE'02 Proceedings of the International Conference on Computers in Education. ACM, pp. 1234-1235 (2002)

22. Sevilla, J., Herrera, G., Martinez, B., Alcantud, F.: Web accessibility for individuals with cognitive deficits: a comparative study between an existing commercial web and its cognitively accessible equivalent. ACM T Comput.-Hum. Int. 14(3) (2007). doi: $10.1145 / 1279700.1279702$

23. Freeman, E., Clare, L., Savitch, N., Royan, L., Litherland, R., Lindsay, M.: Improving website accessibility for people with early-stage dementia: a preliminary investigation. Aging Ment. Health 9, 442-448 (2005)

24. Small, J., Schallau, P., Brown, K., Appleyard, R.: Web accessibility for people with cognitive disabilities. In: Conference on Human Factors in Computing Systems-Proceedings. Association for Computing Machinery, pp. 1793-1796 (2005). doi:10. 1145/1056808.1057024

25. Mahmud, A.A., Martens, J.B.: Understanding email communication of persons with aphasia. In: CHI'11 Proceedings of the SIGCHI Conference on Human Factors in Computing Systems. ACM, pp. 1195-1200 (2011) 
26. Sohlberg, M.M., Ehlhardt, L.A., Fickas, S., Sutcliffe, A.: A pilot study exploring electronic (or e-mail) mail in users with acquired cognitive-linguistic impairments. Brain Inj. 17, 609-629 (2003)

27. Barbieri, T., Bianchi, A., Fraternali, P., Tacchella, C.: AutonoMamente project - design, implementation and evaluation of a multimodal domotic application to support persons with cognitive disabilities. In: 2010 12th IEEE International Conference on e-Health Networking, Applications and Services (Healthcom 2010). IEEE, pp. 324-331 (2010). doi:10.1109/health.2010.5556591

28. Sohlberg, M.M., Fickas, S., Ehlhardt, L.A., Todis, B.: The longitudinal effects of accessible email for individuals with severe cognitive impairments. Aphasiology 19(7), 651-681 (2005)

29. Sesto, M.E., Nelson, R.K., Long, Y., Vanderheiden, G.C.: Evaluation of an experimental mainstream cellular phone feature to allow use by individuals with moderate to severe cognitive disabilities. Univ. Access Inf. Soc. 7, 25-30 (2008). doi:10.1007/ s10209-007-0099-3

30. Stock, S.E., Davies, D.K., Davies, K.R., Wehmeyer, M.L.: Evaluation of an application for making palmtop computers accessible to individuals with intellectual disabilities. J. Intellect. Dev. Disabil. 31, 39-46 (2006)

31. Stock, S.E., Davies, D.K., Wehmeyer, M.L., Palmer, S.B.: Evaluation of cognitively accessible software to increase independent access to cellphone technology for people with intellectual disability. J. Intellect. Disabil. Res. 52, 1155-1164 (2008)

32. Carrasco, E., Epelde, G., Moreno, A., Ortiz, A., Garcia, I., Buiza, C., Urdaneta, E., Etxaniz, A., Gonzalez, M.F., Arruti, A.: Natural interaction between avatars and persons with Alzheimer's disease. In: Computers Helping People with Special Needs. 11th International Conference, ICCHP 2008. Springer, pp. 38-45 (2008). doi:10.1007/978-3-540-70540-6_5

33. Kuwabara, K., Hayashi, S., Uesato, T., Umadome, K., Takenaka, $\mathrm{K}$.: Remote conversation support for people with aphasia: some experiments and lessons learned. In: Universal Access in HumanComputer Interaction. Addressing Diversity. Proceedings 5th International Conference, UAHCI 2009. Springer, pp. 375-384 (2009). doi:10.1007/978-3-642-02707-9_43

34. Keskinen, T., Heimonen, T., Turunen, M., Rajaniemi, J.P., Kauppinen, S.: SymbolChat: a flexible picture-based communication platform for users with intellectual disabilities. Interact. Comput. 24, 374-386 (2012). doi:10.1016/j.intcom.2012.06.003

35. Tuset, P., Barberan, P., Janer, L., Busca, E., Delgado, S., Vila, N.: Messenger visual: A pictogram-based IM service to improve communications among disabled people. In: NordiCHI 2010: Extending Boundaries. Proceedings of the 6th Nordic Conference on Human-Computer Interaction. Association for Computing Machinery, pp. 797-800 (2010). doi:10.1145/1868914. 1869032

36. Stern, P., Shalev, L.: The role of sustained attention and display medium in reading comprehension among adolescents with ADHD and without it. Res. Dev. Disabil. 34, 431-439 (2013). doi:10.1016/j.ridd.2012.08.021

37. Dickinson, A., Gregor, P., Newell, A.F.: Ongoing investigation of the ways in which some of the problems encountered by some dyslexics can be alleviated using computer techniques. In: ASSETS 2002. Proceedings of the Fifth International ACM SIGCAPH Conference on Assistive Technologies. ACM, pp. 97-103 (2002). doi:10.1145/638249.638268

38. Gregor, P., Newell, A.F.: An empirical investigation of ways in which some of the problems encountered by some dyslexics may be alleviated using computer techniques. In: ASSETS'00
Proceedings of the Fourth International ACM Conference on Assistive Technology. ACM (2000)

39. Diraa, N., Engelen, J., Ghesquiere, P., Neyens, K.: The use of ICT to support students with dyslexia. In: Holzinger, A., Miesenberger, K. (eds) Hci and Usability for E-Inclusion, Proceedings, vol. 5889. Lecture Notes in Computer Science, pp. 457-462 (2009)

40. Draffan, E.A., Evans, D.G., Blenkhorn, P.: Use of assistive technology by students with dyslexia in post-secondary education. Disabil. Rehabil. Assist. Technol. 2, 105-116 (2007)

41. Ma, X., Boyd-Graber, J., Nikolova, S., Cook, P.R.: Speaking through pictures: images vs. icons. In: Proceedings of the 11th International ACM SIGACCESS Conference on Computers and Accessibility. ACM, pp. 163-170 (2009)

42. Beacham, N.A., Alty, J.L.: An investigation into the effects that digital media can have on the learning outcomes of individuals who have dyslexia. Comput. Educ. 47, 74-93 (2006)

43. Grynszpan, O., Martin, J.C., Nadel, J.: Multimedia interfaces for users with high functioning autism: an empirical investigation. Int. J. Hum. Comput. Stud. 66(8), 628-639 (2008). doi:10.1016/j. ijhcs.2008.04.001

44. Zentel, P., Opfermann, M., Krewinkel, J.: Multimedia learning and the Internet: ensuring accessibility for people with learning disabilities. J. Assist. Technol. 1, 22-32 (2007)

45. Jimison, H.B., Sher, P.P., Appleyard, R., LeVernois, Y.: The use of multimedia in the informed consent process. J. Am. Med. Inform. Assoc. 5, 245-256 (1998)

46. Kvale, K., Warakagoda, N.: A speech centric mobile multimodal service useful for dyslectics and aphasics. In: 9th European Conference on Speech Communication and Technology. International Speech and Communication Association, pp. 461-464 (2005)

47. Vigouroux, N., Rumeau, P., Vella, F., Vellas, B.: Studying pointselect-drag interaction techniques for older people with cognitive impairment. In: Universal Access in Human-Computer Interaction. Addressing Diversity. Proceedings 5th International Conference, UAHCI 2009. Springer, pp. 422-428 (2009). doi:10. 1007/978-3-642-02707-9_48

48. Bunning, K., Kwiatkowska, G., Weldin, N.: People with profound and multiple intellectual disabilities using symbols to control a computer: exploration of user engagement and supporter facilitation. Assist. Technol. 24, 259-270 (2012). doi:10.1080/ 10400435.2012.659832

49. Sharp, H., Rogers, Y., Preece, J.: Interaction Design: Beyond Human-Computer Interaction, 2nd edn. Wiley, West Sussex (2007)

50. Richardson, A.: Those WCAG forgot: designing for the cognitively disabled. Orange J. Tech. Commun. Inf. Des. 7(2). http:// orange.eserver.org/issues/7-2/richardson.html (2011). Accessed 18 Sept 2013

51. W3C: Web Content Accessibility Guidelines (WCAG) 2.0. http:// www.w3.org/TR/WCAG20/ (2008). Accessed 18 Sept 2013

52. WHO: International Statistical Classification of Diseases and Related Health Problems, Tenth Revision, Vols. 1-3. World Health Organization, Geneva (1994)

53. APA: Quick Reference to the Diagnostic Criteria from DSM-IVTR. American Psychiatric Association, Washington (2002)

54. AEGIS: About AEGIS. http://www.aegis-project.eu (2014). Accessed 14 Jan 2014

55. Cloud4all: What is Cloud4all? http://www.cloud4all.info (2014). Accessed 14 Jan 2014 